\title{
COVID-19 and COPD: a narrative review of the basic science and clinical outcomes
}

\author{
Andrew Higham (1) ${ }^{1}$, Alexander Mathioudakis (1) ${ }^{1}$, Jørgen Vestbo (i) ${ }^{1}$ and \\ Dave Singh ${ }^{1,2}$
}

Affiliations: 'Division of Infection, Immunity and Respiratory Medicine, School of Biological Sciences, Faculty of Biology, Medicine and Health, Manchester Academic Health Science Centre, The University of Manchester and Manchester University NHS Foundation Trust, Manchester, UK. ${ }^{2}$ Medicines Evaluation Unit, Manchester, UK.

Correspondence: Andrew Higham, University of Manchester, 2nd Floor ERC, Southmoor Road, Manchester, M23 9LT, UK. E-mail: Andrew.Highamamanchester.ac.uk

@ERSpublications

This up-to-date review tackles some of the key issues which have significant impact on the long-term outlook for COPD patients in the context of COVID-19 https://bit.ly/36PKzEO

Cite this article as: Higham A, Mathioudakis A, Vestbo J, et al. COVID-19 and COPD: a narrative review of the basic science and clinical outcomes. Eur Respir Rev 2020; 29: 200199 [https://doi.org/10.1183/ 16000617.0199-2020].

ABSTRACT The 2019 coronavirus disease (COVID-19) pandemic is caused by severe acute respiratory syndrome-coronavirus-2 (SARS-CoV-2). Clinical outcomes, including mortality, are worse in males, older individuals and patients with comorbidities. COPD patients are included in shielding strategies due to their susceptibility to virus-induced exacerbations, compromised pulmonary function and high prevalence of associated comorbidities. Using evidence from basic science and cohort studies, this review addresses key questions concerning COVID-19 and COPD. First, are there mechanisms by which COPD patients are more susceptible to SARS-CoV-2 infection? Secondly, do inhaled corticosteroids offer protection against COVID-19? And, thirdly, what is the evidence regarding clinical outcomes from COVID-19 in COPD patients? This up-to-date review tackles some of the key issues which have significant impact on the longterm outlook for COPD patients in the context of COVID-19.

\section{Introduction}

Severe acute respiratory syndrome-coronavirus-2 (SARS-CoV-2) is responsible for the global coronavirus disease (COVID-19) pandemic. SARS-CoV-2 is a beta coronavirus believed to have originated in the city of Wuhan, China [1]. SARS-CoV-2 may cause asymptomatic infection or a mild viral illness, while more severe COVID-19 cases are characterised by high fever, cough and dyspnoea [2-4]. Other commonly reported symptoms include myalgia, fatigue, gastro-intestinal disturbance, anosmia and sputum production [2-4]. A proportion of patients with COVID-19 develop pneumonia and acute severe respiratory failure, which is associated with a high mortality $[4,5]$. A feature of severe COVID-19 is high levels of systemic inflammation, the so called "cytokine storm" [6].

Acute severe respiratory failure associated with COVID-19 is characterised by severe hypoxaemia with good lung compliance [7]. This suggests vascular injury and/or vasoconstriction are key underlying causes for respiratory failure, with microvascular injury causing the leaky pulmonary exudate typical of COVID-19 pneumonia. Additionally, severe COVID-19 patients show abnormal levels of systemic pro-coagulation markers including high $\mathrm{D}$-dimer levels and low platelet counts, implicating pulmonary thrombosis as a contributor to respiratory failure $[4,8]$. Autopsies have confirmed that typical pathological

Provenance: Submitted article, peer reviewed

Received: 22 June 2020 | Accepted after revision: 2 Oct 2020

Copyright CERS 2020. This article is open access and distributed under the terms of the Creative Commons Attribution Non-Commercial Licence 4.0. 
features of severe COVID-19 include endothelial injury and thrombotic microangiopathy [9, 10]. Multi-organ involvement in severe COVID-19 is common, including renal disease and neurological involvement, suggesting that endothelial disease and microvascular involvement are central pathophysiological processes in COVID-19 [3, 4, 8, 11].

Clinical outcomes, including mortality, in COVID-19 are worse in males, older individuals and patients with diabetes, cardiovascular disease and obesity $[3-5,11,12]$. This information has been used to guide "shielding" strategies during the COVID-19 pandemic, identifying high-risk sub-groups who should remain at home, away from social contact that allows viral transmission. COPD patients have been included in this shielding strategy due to their susceptibility to virus-induced exacerbations, compromised pulmonary function and high prevalence of associated comorbidities [13-17]. This narrative review sets out to address key questions concerning COVID-19 and COPD. First, are there mechanisms causing COPD patients to be more susceptible to SARS-CoV-2 infection? Secondly, do inhaled corticosteroids (ICS) offer protection against COVID-19? And, finally, what is the evidence regarding clinical outcomes from COVID-19 in COPD patients?

\section{Mechanisms of susceptibility to SARS-CoV-2 infection in COPD patients}

Entry of SARS-CoV-2 into host cells is a sequential process involving cellular attachment and endocytosis [18]. This is mediated by the membrane bound viral spike protein which consists of the S1 receptor binding subunit, and the S2 membrane fusion subunit. In common with other coronaviruses, SARS-CoV-2 uses angiotensin converting enzyme 2 (ACE2) as a receptor for cellular attachment (mediated by the S1 subunit) $[19,20]$. It is thought that SARS-CoV-2 spike protein mutations enable greater affinity for binding to ACE2, thereby enhancing the ability of this virus to gain cellular entry [21].

ACE2 is a transmembrane peptidase which hydrolyses angiotensin II to produce angiotensin 1-7 [22]. Angiotensin II acts directly on vascular smooth muscle cells through the angiotensin type 1 (AT1) receptor to cause cellular contraction, and thus increased vascular tone [23]. Intravenous infusion of angiotensin II increases pulmonary vascular pressure in human subjects [24]. Sustained high pulmonary vascular pressures cause hydrostatic oedema due to leakage from the single cell thick capillary bed [25]. Angiotensin II also increases microvascular permeability; a study using rat post capillary venules showed increased fluid movement across the endothelial layer in response to angiotensin II [26].

ACE2 is expressed throughout the body, including in the lungs, where expression has been confirmed in the trachea, large airway epithelium, small airway epithelium, type 2 pneumocytes and endothelium [27-30]. ACE2 has a homeostatic protective role in the lungs by limiting the effects of angiotensin II activity on vascular tone and permeability and increasing the production of angiotensin 1-7 which has vasodilator activity. Angiotensin II also causes pro-inflammatory cytokine production [31]. Reduced ACE2 activity resulted in increased pulmonary cytokine levels and neutrophil influx in endotoxin exposed mice, coupled with increased vascular permeability and lung oedema [32]. In contrast, angiotensin 1-7 reduces experimental lung injury in rats [33]. A loss of ACE2 function may therefore enhance host inflammatory responses and cause vasoconstriction and vascular injury.

In vitro studies have confirmed that a lack of ACE2 expression prevents SARS-CoV-2 infection [19]. Furthermore, the degree of SARS-CoV infection of epithelial cells is related to the level of ACE2 expression [34]. Emerging evidence from computational studies suggests genetic variants of ACE2 structure may alter SARS-CoV-2 interaction thereby increasing susceptibility to infection [35]. However, it is the degree to which altered ACE2 expression levels or genetic variation causes increased susceptibility to SARS-CoV-2 infection in humans or the development of severe COVID-19 that remains unclear. The role of ACE2 in the disease process is likely part of a complex and multi-factorial sequence of pathophysiological mechanisms [36].

During SARS-CoV-2 infection, cell surface ACE2 activity may be reduced due to internalisation or shedding, following binding of the virus, as demonstrated with SARS-CoV infection [37]. Interestingly, SARS-CoV, but not HCoV NL63, causes ACE2 shedding and increased lung injury in murine models, with concurrent increases in angiotensin II levels [38]. Importantly, AT1 receptor antagonism attenuated these affects [39]. Collectively, these observations implicate reduced ACE2 activity in COVID-19 lung injury (figure 1).

The second phase of SARS-CoV-2 cellular entry is fusion between the host and virus membranes, where the spike protein undergoes proteolytic cleavage at the S1/S2 interface which facilitates fusion between the virus and host membranes (mediated by the S2 subunit) and subsequent cell entry [18, 20]. The virus utilises host proteases including furin, transmembrane serine protease 2 (TMPRSS2) and cathepsins during this process $[18,20]$. 


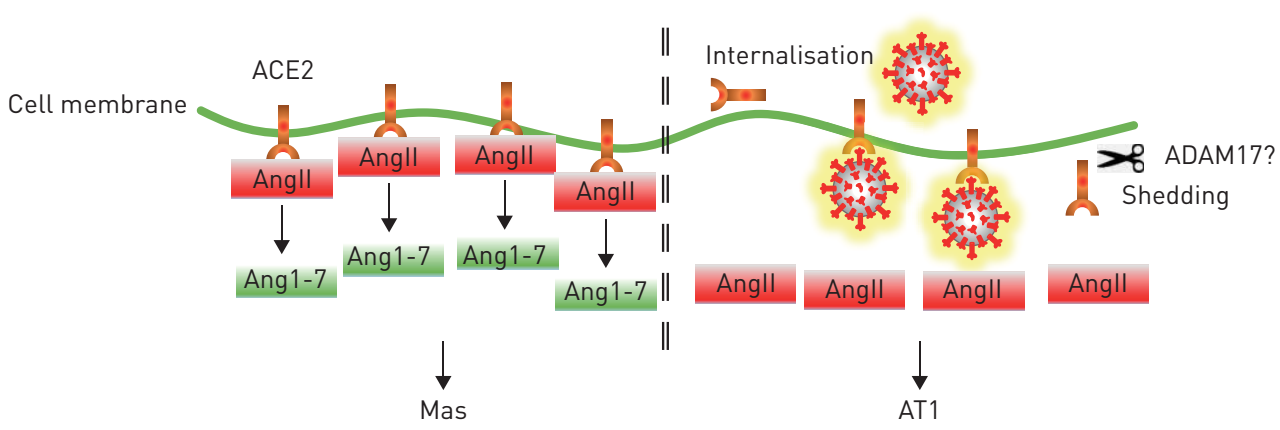

Anti-inflammatory

Pro-inflammatory Vascular permeability Lung injury Vasodilation Vasoconstriction Oedema

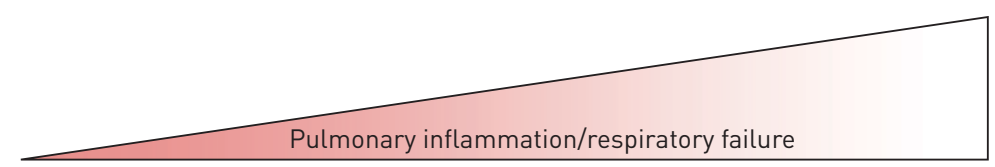

No infection
ACE2 working at capacity and
Angll levels under control

SARS-CoV-2 mediated injury

ACE2 activity reduced and Angll levels increased

FIGURE 1 The implications of angiotensin converting enzyme (ACE)2 dysfunction during severe acute respiratory syndrome-coronavirus-2 (SARS-CoV-2) infection. In the absence of infection, ACE2 is working at capacity and the levels of angiotensin II (ang II) are tightly regulated by conversion to angiotensin 1-7 (ang 1-7). Ang 1-7 activates the Mas receptor to regulate inflammation and vasomotor tone. During SARS-CoV-2 infection, ACE2 activity is reduced due to receptor occupancy, shedding and internalisation and the levels of ang II increase. Ang II activates the AT1 receptor to cause increased pro-inflammatory cytokine production, increased vasoconstriction, increased vascular permeability, oedema and lung injury. Pulmonary inflammation increases and acute severe respiratory failure may ensue.

Recent gene and protein expression studies have demonstrated increased ACE2 expression in the bronchial epithelium and whole lung tissue of COPD patients compared to controls, with an association between higher ACE2 expression levels and lower lung function (table 1). LEung et al. [28] used bronchial brushing samples obtained by bronchoscopy to demonstrate increased ACE2 gene expression in COPD patients compared to controls, which included a mixture of never-, former and current smokers. In these samples, current smokers had higher ACE2 gene expression levels. The authors also used immunohistochemistry to

TABLE 1 Expression of genes/proteins related to severe acute respiratory syndrome-coronavirus-2 infection in controls ${ }^{\#}$ and COPD patients

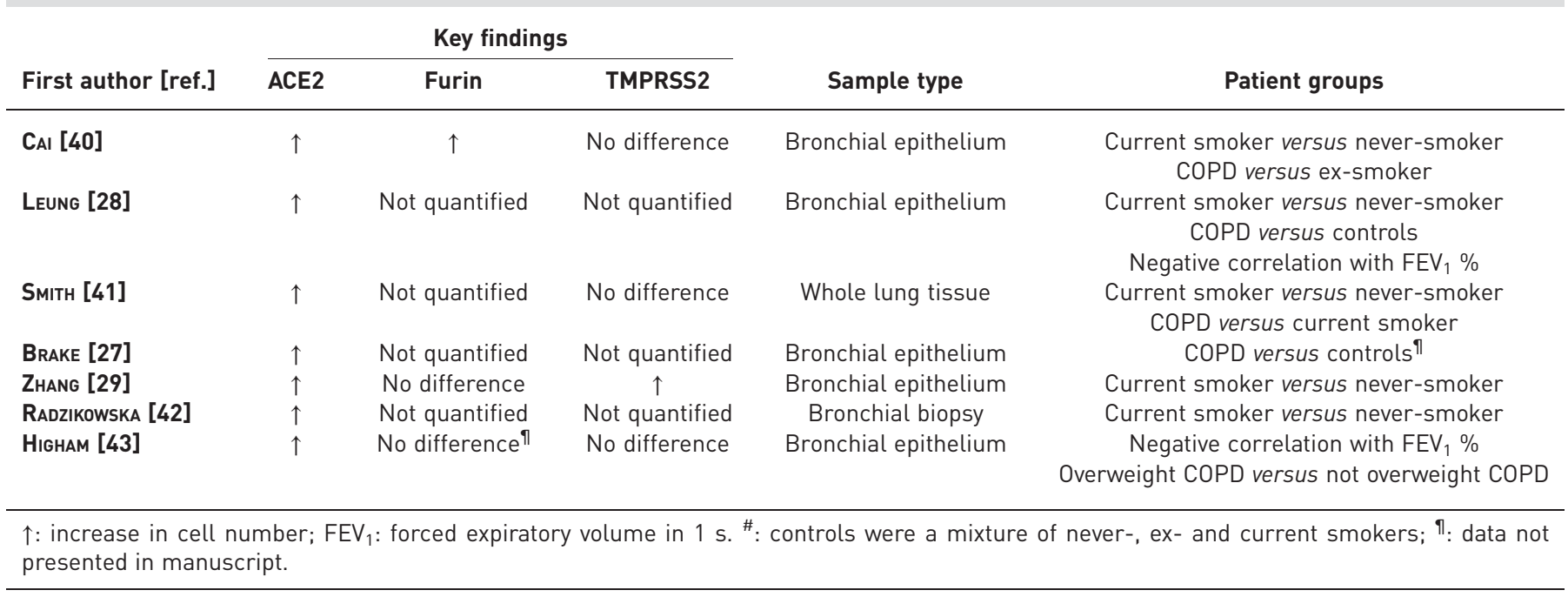


show increased ACE2 protein expression in the small airway epithelium of COPD compared to never-smokers but not current smokers. CAI et al. [40] observed increased ACE2 gene expression in bronchial brushing samples of COPD patients compared to former smokers but not current smokers. SMITH et al. [41] demonstrated ACE2 expression was significantly higher in the whole lung tissue of COPD patients compared to smokers. RADZIKOWSKA et al. [42] observed increased ACE2 gene expression in bronchial biopsies of smokers compared to never-smokers but no difference in COPD versus controls, however the number of COPD samples were small $(n=3)$. The overall pattern of these data is that COPD patients have increased ACE2 expression compared to never-smoker controls, and that smoking itself upregulates ACE2 expression. Increased ACE2 expression in COPD patients compared to smokers (without airflow obstruction) was less consistent; differences between studies may be related to site of sampling, analytical methodology, or number of samples.

Cigarette smoke exposure increases ACE2 gene expression in mice [41]. In primary bronchial epithelial cells, nicotine dependent activation of the $\alpha 7$ subtype of nicotine acetylcholine receptors ( $\alpha 7-\mathrm{nAChR})$ was shown to increase ACE2 gene expression [44]. The levels of CHRNA7 (the gene which encodes $\alpha 7-n A C h R)$ in the bronchial epithelium are higher in current smokers and levels positively correlate with ACE2 expression $\left(\mathrm{r}=0.54 \mathrm{p}=2.31 \times 10^{-8}\right)$ and negatively correlate with forced expiratory volume in $1 \mathrm{~s}$ $\left(\mathrm{FEV}_{1}\right) \%$ predicted $\left(\mathrm{r}=-0.37 \mathrm{p}=2.83 \times 10^{-4}\right)$ in COPD patients [45]. We have been able to replicate these findings concerning CHRNA7 using a cohort of 37 COPD patients (correlation with ACE2: $\mathrm{r}=0.4 \mathrm{p}=0.02$; correlation with $\mathrm{FEV}_{1} \%: \mathrm{r}=-0.4 \mathrm{p}=0.02$ previously unpublished data).

Using single cell RNA sequencing, enabling identification of specific cell types in a mixed cell population, it was shown that several epithelial cell types including basal cells, intermediate cells, ciliated cells and secretory cells (goblet/club) express ACE2 [29, 41, 46, 47]. Differences exist in the conclusions of these studies, with some suggesting ACE2 expression was particularly high in secretory cells [41, 46]. The location of sampling, donor variability and methodology used to analyse expression may explain these differences. Nevertheless, it is important to recognise that epithelial remodelling as a result of injury and repair may impact epithelial cell phenotype, including ACE2 expression. Cigarette smoking is a key driver of goblet cell hyperplasia, and goblet cell numbers are increased in COPD airways [48]. Furthermore, $\alpha 7$-nAChR regulates mucous production in bronchial epithelial cells exposed to nicotine [49]. Cigarette smoke, and in particular nicotine, dependent activation of $\alpha 7-\mathrm{nAChR}$ may therefore drive concurrent goblet cell hyperplasia and increased ACE2 expression in the airways of COPD patients.

We have shown increased gene expression of ACE2 in the bronchial epithelium of COPD patients who are overweight (mean body mass index (BMI) $29 \mathrm{~kg} \cdot \mathrm{m}^{-2}$ ) compared to those not overweight (mean BMI $23 \mathrm{~kg} \cdot \mathrm{m}^{-2}$ ) [43]. This suggests comorbidities, or even diet, may regulate expression of ACE2 in the lungs. This is supported by observations showing increased ACE2 expression in the adipose tissue of individuals with obesity [50].

CAI et al. [40] demonstrated increased gene expression of furin, but not TMPRSS2, in the bronchial epithelium of smokers compared to never-smokers, but found no difference in furin or TMPRSS2 expression in COPD patients compared to controls. In contrast, ZHANG et al. [29] reported increased gene expression of TMPSSR2, but not furin, in the bronchial epithelium of smokers compared to never-smokers, while COPD patients were not studied. Whilst the results are not consistent, it appears cigarette smoking may alter protease expression, but modulation of expression in COPD is less clear.

These recent studies investigating susceptibility to SARS-CoV-2 infection in COPD patients have focused on ACE2 and protease expression. However, it should also be remembered that COPD patients have increased susceptibility generally to viral infections, possibly due to decreased type 1 interferon (IFN) production [51] or immunosenescence, characterised by increased numbers of exhausted T-cells and reduced numbers of memory T-cells [52-54]. Any increase in ACE2 levels in COPD patients, thereby increasing susceptibility to SARS-CoV-2 infection, therefore occurs on a background of suboptimal host defence.

Evidence of endothelial cell dysfunction and coagulopathy have been reported in COPD patients. The number of apoptotic endothelial cells is increased in COPD patients and increased permeability of the airway microvasculature in COPD patients is related to the degree of airflow limitation [55, 56]. Circulating levels of pro-coagulation factors are increased in COPD patients, which increase further during exacerbations $[57,58]$. This likely contributes to the occurrence of pulmonary embolisms that are reported in COPD patients with exacerbations [59]. COPD patients may therefore be more susceptible to vascular damage and thrombosis during SARS-CoV-2 infection.

The available evidence suggests COPD patients may be more susceptible to SARS-CoV-2 infection due to changes in ACE2 expression. Cigarette smoking appears to be an important risk factor, whilst preliminary 
evidence suggests that obesity may play a role. Increased susceptibility to vascular abnormalities may also be involved.

\section{ICS use in COPD: implications for COVID-19}

An exacerbation is defined as a worsening of COPD symptoms resulting in the need for additional pharmacological treatment [60]. COPD patients with more frequent exacerbations suffer with worse clinical outcomes, including lung function decline and mortality [61, 62]. Viral infections are a common cause of COPD exacerbations $[13,14,16,17]$, with secondary bacterial infections commonly occurring $[14,63]$.

Multiple randomised clinical trials have shown that ICS reduce exacerbation rates when used as part of a combination treatment with a long-acting $\beta$-agonist (LABA) or a LABA plus a long-acting muscarinic antagonist [64-66]. This ICS benefit appears to be greater in patients with higher blood eosinophil counts, with little or no benefit in patients with lower counts $\left(<100\right.$ eosinophils. $\left.\mu \mathrm{L}^{-1}\right)$ [67-71]. ICS treatment can cause side-effects, including osteoporosis, diabetes and most notably pneumonia $[72,73]$. Due to these potential risks, it is recommended that ICS are used in a personalised manner using exacerbation risk and blood eosinophil counts to identify individuals most likely to benefit [74]. The association between eosinophil counts and clinical benefit suggests that ICS target type 2 inflammation in COPD, as is the case in asthma.

Corticosteroids suppress pro-inflammatory cytokine production from various cell types, through trans-repression of gene transcription $[75,76]$. Bronchoscopy and sputum sampling studies have shown that ICS treatment can reduce inflammatory cell counts in the lungs [77, 78]. While these anti-inflammatory effects provide protection against COPD exacerbations, there are also molecular mechanisms whereby corticosteroids may increase susceptibility to infection. The suppression of innate immune cytokines may impair the ability of the host defence to counter bacterial infection. The reported association between ICS treatment and increased presence of colonising bacteria supports this possibility $[79,80]$. The phagocytosis ability of alveolar macrophages is reduced in COPD patients [81], but we have recently demonstrated that corticosteroids do not further suppress phagocytosis by these cells [82]. Interestingly, a recent longitudinal cohort study showed that ICS use increased the risk of pneumonia in COPD patients with chronic bacterial infection or low blood eosinophil counts $\left(<100\right.$ eosinophils $\left.\mu \mathrm{L}^{-1}\right)$ [83]. The mechanism for the association between ICS and both increased bacterial presence and increased pneumonia incidence has yet to be conclusively elucidated.

Corticosteroids suppress the production of the anti-viral type I and III IFN from epithelial cells [85, 86]. This is associated with increased viral replication, and excess mucin production [84]. The use of ICS in COPD patients may therefore increase susceptibility to virus infection, and/or worsen clinical outcomes through these mechanisms [87]. Secondary bacterial infection in such cases provide one possible explanation for the increased bacterial presence and pneumonia risk observed with ICS use in COPD patients [83]. Overall, the benefits of ICS appear to outweigh these infection risks in patients with higher eosinophil counts, but in those with lower eosinophil counts the benefit-risk ratio often does not justify the use of these drugs [74].

There is in vitro evidence that bronchial epithelial cells treated with the corticosteroid budesonide, in combination with the bronchodilators glycopyrronium and formoterol, inhibits HCoV-229E replication [88]. The corticosteroid ciclesonide also appears to attenuate SARS-CoV-2 replication in vitro by targeting non-structural protein 15, an endoribonuclease which helps evade host detection of viral double-stranded RNA and type 1 IFN responses [89-91]. ICS may also prevent SARS-CoV-2 entry; ACE2 gene expression is lower in the sputum of COPD and asthma patients who use ICS compared to those who do not [92, 93]. Furthermore, studies in mice have shown that ICS reduce ACE2 expression by inhibiting type 1 IFN production [92]. While suppression of type 1 IFN secretion may reduce host defence, the associated reduction of ACE2 expression may protect against SARS-CoV-2 cellular entry. These findings raise the possibility that ICS use in COPD patients may protect against COVID-19 (figure 2).

A systematic literature review did not find any studies that could determine whether ICS are associated with better or worse clinical outcomes in COPD patients with severe coronavirus infections including COVID-19, SARS and Middle East Respiratory Syndrome [94]. Recently, observational data taken from UK electronic health records reported increased COVID-19 associated mortality in COPD and asthma patients using ICS compared to those not using ICS [95]. However, a sensitivity analysis also showed increased mortality due to COVID-19 in COPD patients treated with ICS plus two long-acting bronchodilators (triple therapy) compared to ICS plus one long-acting bronchodilator, indicating a confounding effect of COPD due to greater disease severity in patients treated with triple therapy that was not due to ICS itself. A negative control analysis also highlighted more non-COVID-19 deaths in patients 


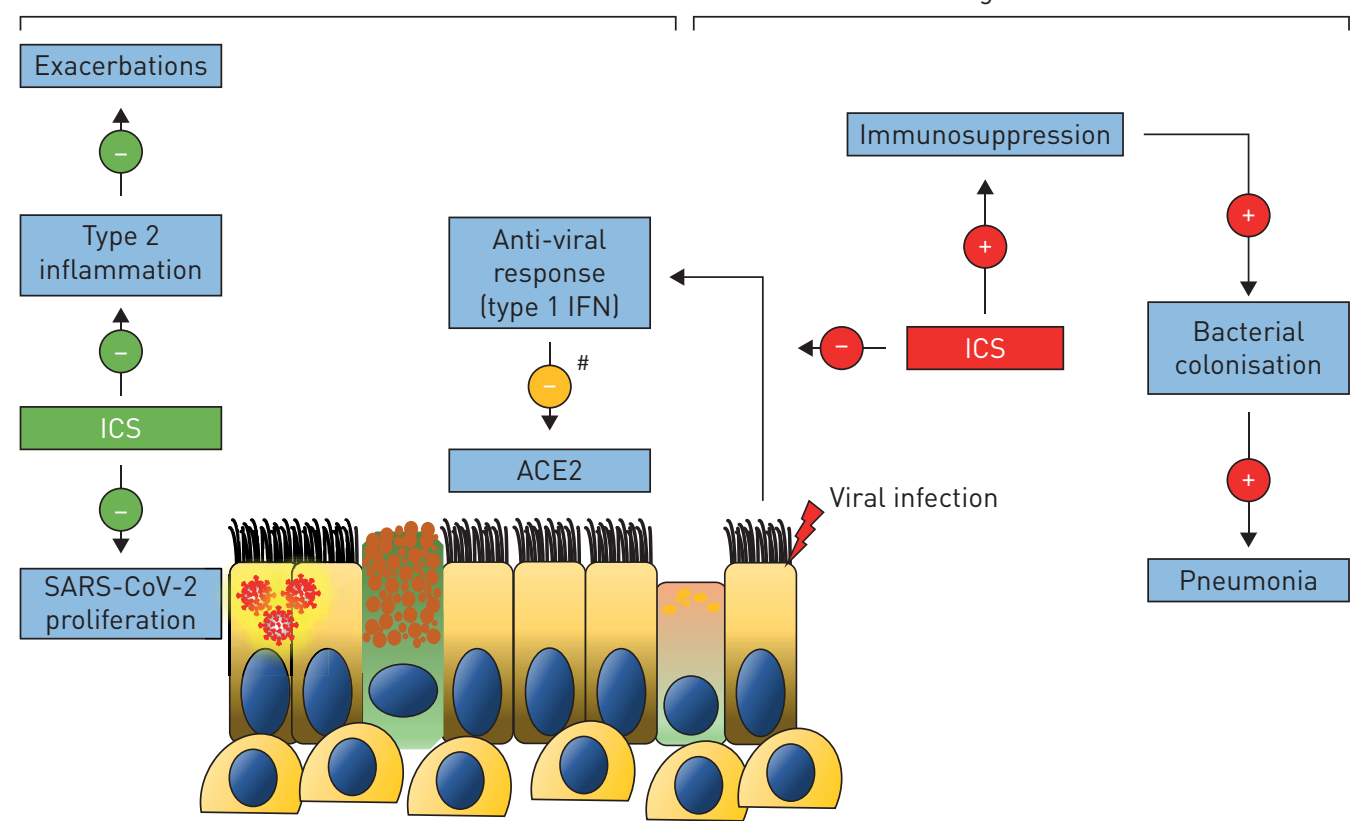

FIGURE 2 Inhaled corticosteroid (ICS) use in COPD: implications for coronavirus disease 2019. ICS prevent exacerbations in eosinophilic COPD patients, probably in part by targeting type 2 inflammation in these individuals. ICS may have further benefit by reducing the ability of severe acute respiratory syndrome-coronavirus-2 (SARS-CoV-2) to proliferate, and by limiting SARS-CoV-2 cellular entry by reducing angiotensin converting enzyme (ACE)2 expression as a result of inhibiting type 1 interferon (IFN) production. However, immunosuppression may increase susceptibility to respiratory infections leading to secondary bacterial colonisation and increasing the risk for pneumonia in some individuals. ": ICS reduces ACE2 expression by reducing type 1 IFN production.

treated with ICS, further re-enforcing confounding by disease severity. This analysis highlights the practical issues regarding real-life data collection with regard to understanding whether ICS use has a protective or detrimental effect in COPD patients with regard to COVID-19. In the absence of randomised controlled trial data, COPD patients are being advised to continue with their usual inhaled treatment regime, including ICS if already being used [94]. COPD management guidelines provide recommendations regarding clinical situations where it is appropriate to withdraw ICS treatment [96]. Appropriate ICS withdrawal should continue to be considered at the current time, in the absence of conclusive data showing that ICS use has a protective effect against COVID-19 in COPD patients.

\section{Epidemiology and clinical outcomes of COVID-19 in COPD patients}

A number of publications have evaluated the epidemiology, clinical characteristics and clinical outcomes of COVID-19. There is significant diversity in the clinical settings of these studies, and the type of data collected. Accordingly, the case fatality rates of patients with severe (hospitalised) COVID-19 disease vary from $1 \%$ to $62 \%$ [97]. Data on the epidemiology and outcomes of COVID-19 among patients with COPD are still being accumulated. Here, we review the distinct issues of whether COPD is associated with an increased risk of acquiring COVID-19, or an increased risk of worse outcomes with COVID-19.

The prevalence of COPD among patients with COVID-19 was summarised in a systematic review of 15 studies that had been published by 24th March 2020 involving 2473 patients with confirmed COVID-19, mainly from China; a prevalence of $2 \%$ (95\% CI 1-3\%) was reported (figure 3) [98]. A similar prevalence (3\%), was found in a more recent report assessing 13442 patients diagnosed with COVID-19 after an emergency department visit or admission in New York (NY, USA) [101] and a report of 1099 unselected patients from China $(1 \%, 95 \%$ CI $0.6-1.9 \%)$ [100]. To explain the lower than anticipated observed prevalence of COPD among patients with COVID-19, it has been proposed that either the disease or its treatment may reduce the risk of infection [108]. None of these hypotheses have been proven yet. COPD under-diagnosis is a well-known issue that may contribute to these findings [108], or that full information regarding comorbidities including COPD was not recorded in the clinical notes. It is also likely that the low observed prevalence of COPD in some populations results predominantly from the early shielding of older and high-risk populations, including patients with COPD, in many countries [109]. This is supported by the higher prevalence of COPD (6.6\%) and the higher median age (63 years compared to 


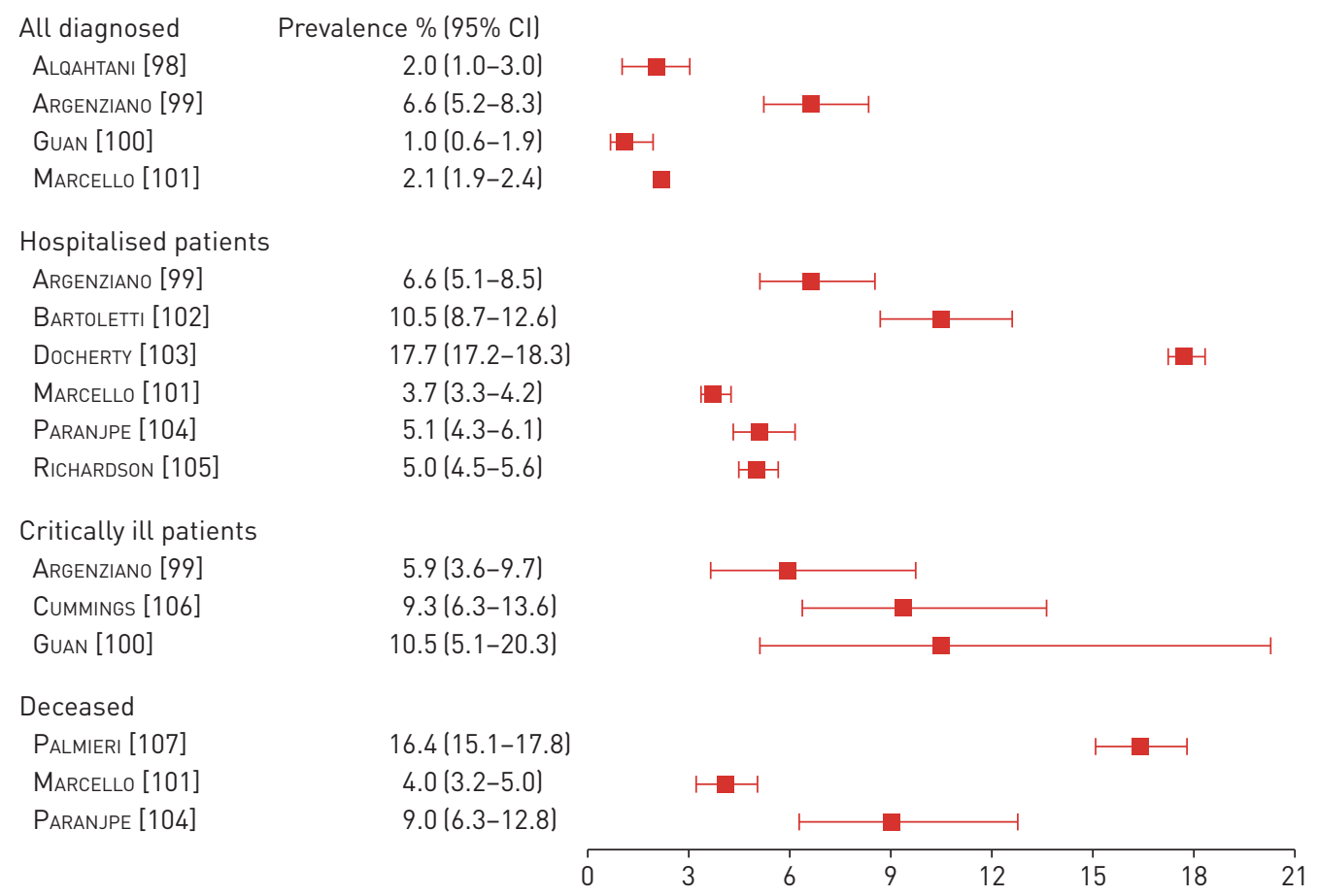

FIGURE 3 Prevalence of COPD among patients with coronavirus disease 2019 with different severity. Data summary from larger patient cohorts ( $n>1000$ for hospitalised patients or $>500$ for critically ill patients).

52.7 years) that were found in a case series of 1000 consecutive patients diagnosed with COVID-19 early in the course of the epidemic in New York [99]. Nevertheless, none of these observations provide definitive information demonstrating that patients with COPD have an increased risk of acquiring SARS-CoV-2 infection or developing COVID-19.

An age- and sex-adjusted meta-analysis of 11 case series in China and the USA suggested that current cigarette smoking might be protective against contracting COVID-19; the prevalence of current smokers among patients was significantly lower than anticipated (prevalence OR 0.20, 95\% CI 0.13-0.31) [110]. However, current smokers have higher prevalence of cardiovascular and respiratory diseases and were therefore more likely to be shielding during the COVID-19 epidemic, introducing a significant unaddressed confounding in the meta-analysis. In addition, most of the COVID-19 series are based on routinely collected data from case records, where current smoking information may be significantly underestimated. A possible reason for this is that information not critical in guiding clinical decisions may have been omitted due to the healthcare burden posed by the ongoing pandemic.

Patients with COPD, as well as current smokers, are consistently reported to have worse outcomes after COVID-19 infection. Several large patient cohorts reported an association between co-existing COPD and worse clinical outcomes among COVID-19 patients in hospitals (figure 4) [99-101, 104-106 111]. The previously mentioned meta-analysis reported an $88 \%$ increased risk of intensive care admission or death among those with co-existing COPD (RR 1.88, 95\% CI 1.4-2.4) [98]. In addition, the risk of developing severe complications was 45\% higher among current smokers (RR 1.45, 95\% CI 1.03-2.04), arguing against a protective effect of current smoking against COVID-19. However, mortality estimates in this meta-analysis are limited by the sample size. More specifically, mortality within the subgroup of patients with co-existing COPD was only reported in two studies involving 10 patients with COPD. Among these patients six died. In the larger New York cohort (13442 patients with COVID-19 attending the emergency department), COPD was associated with an increased risk of hospitalisation (RR 1.77, 95\% CI 1.67-1.87) [100], and a trend for increased mortality (RR 1.08, 95\% CI 0.88-1.33). Similar findings were reported in an Italian cohort involving 1044 hospitalised patients; patients with COPD had significantly increased risk of severe respiratory failure (RR 1.17, 95\% CI 1.09-1.27) [102]. In a Spanish longitudinal cohort, COPD was also associated with a $70 \%$ increase in the risk of death (RR 1.69, 95\% CI 1.23-2.32) [111]. The ISARIC (International Severe Acute Respiratory and Emerging Infection Consortium) cohort, based on data from over 20000 patients hospitalised with COVID-19 infection, demonstrated that non-asthma chronic pulmonary diseases are associated with an increased risk of death (HR 1.17, 95\% CI 1.09-1.27) [103]. 


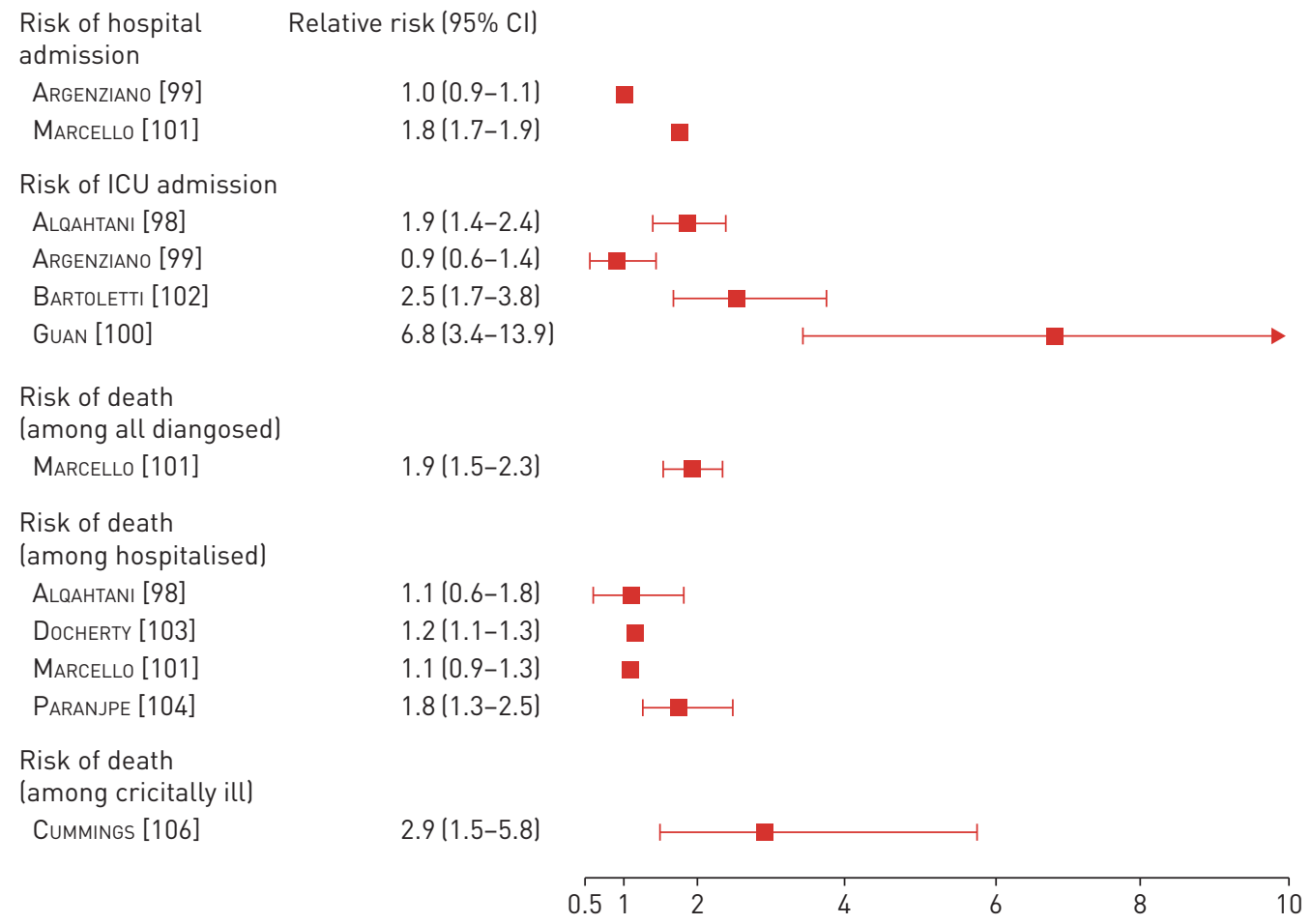

FIGURE 4 Impact of COPD on the outcomes of coronavirus disease 2019. Data summary from larger patient cohorts (n >1000 for hospitalised patients or $>500$ for critically ill patients). ICU: intensive care unit.

Consistently, the prevalence of COPD rises further among patients suffering from more critical or lethal COVID-19 disease. A series of 257 critically ill patients admitted to the intensive care unit with COVID-19 in New York revealed a much higher prevalence of COPD (9\%) and current or former smokers (33\%) [106]. Moreover, COPD was predictive of significantly higher risk of death in univariate regression analyses (HR 3.15, 95\% CI 1.84-5.39), and this association remained robust in multivariate regression (HR 2.94, 95\% CI 1.48-5.84). Interestingly, COPD has been reported as a comorbidity in as many as $16.4 \%$ of patients who did not survive the COVID-19 infection, in an Italian survey involving 3032 patients [107]. This corresponded to a prevalence of $17.2 \%$ among patients aged $\geqslant 65$ years, and $11.1 \%$ among younger patients.

\section{Clinical and research implications}

Risk factors for worse outcomes from COVID-19 include increasing age and cardiovascular comorbidities [2-4]. COPD is a disease that occurs in later life, and is associated with multiple comorbidities including cardiovascular diseases [15]. In addition to the risk conferred by age and comorbidities, the evidence indicates that COPD itself is associated with worse outcomes [99-101, 104-106, 111]. The reasons for this may be increased susceptibility to viral infection (through decreased anti-viral defence or increased ACE2 expression) in COPD or pre-existing compromised pulmonary function. The available data does not deal with the heterogeneity of COPD, including disease severity, exacerbation frequency and comorbidities. Future analysis should consider these features as additional susceptibility factors.

Thrombosis and coagulopathies are common features of severe COVID-19, and COPD patients also demonstrate increased susceptibility to these vascular events [55-59]. It is important to understand if pre-existing endothelial dysfunction in COPD patients predisposes to vascular complications during COVID-19. Future studies should examine how COPD pulmonary endothelial cells behave during infection and inflammation and if this may lead to vascular complications in the micro-circulation.

Evidence is still lacking on the long-term sequelae of COVID-19 among patients with pre-existing respiratory diseases, such as COPD. Emerging evidence from COVID-19 convalescent patients without pulmonary disease shows reduced lung function and computed tomography abnormalities up to 3 months after discharge $[112,113]$. COPD patients demonstrate abnormal remodelling processes following lung injury, so one can expect significantly abnormal tissue remodelling following SARS-CoV-2 infection. Future studies should attempt to understand the impact of SARS-CoV-2 infection on disease pathophysiology including small airway disease and emphysema. 

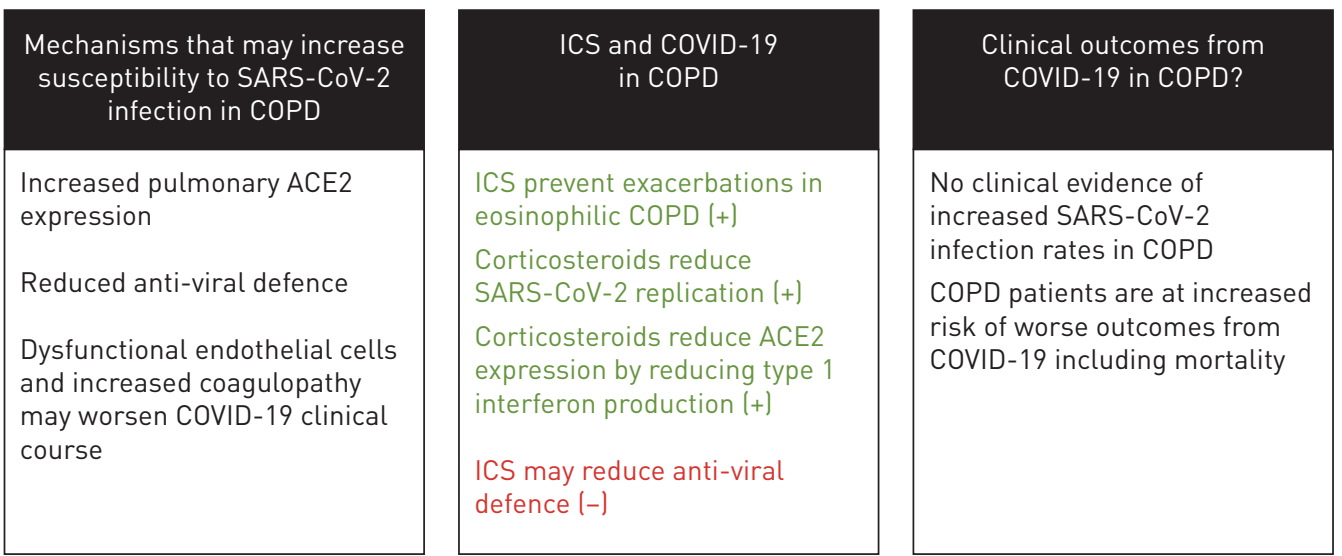

FIGURE 5 The key issues addressed by this review. SARS-CoV-2: severe acute respiratory syndrome-coronavirus-2; COVID-19: coronavirus disease 2019; ICS: inhaled corticosteroids; ACE2: angiotensin converting enzyme 2 . +: benefits of ICS; -: dangers of ICS.

The relationship between ICS use and SARS-CoV-2 infection is still unclear. Whilst there may be some benefit on reducing the ability of SARS-CoV-2 to proliferate $[89,90]$, it is known that ICS can dampen important anti-viral mechanisms [84-86]. Despite dampening anti-viral mechanisms, ICS use in COPD patients prevents exacerbations in patients with higher blood eosinophils [67-70]. Conversely, ICS use in COPD patients with low blood eosinophils counts or chronic bacterial infection can increase the risk of pneumonia [83]. Similarly, ICS use in COPD patients with regard to SARS-CoV-2 infection may be a double edged sword, conferring benefit to some but harm to others. This topic urgently needs further research to support clinical decision making.

The long-term impact of isolation on the natural history of COPD is unclear; this may lead to reduced viral infections in the short term, but may cause undesirable effects on the general physical and psychosocial health of these patients. The resulting decrease in physical activities and exercise may deprive patients of the beneficial effects that include improved quality of life, decreased symptoms burden and risk of exacerbations and mortality [60].

We recognise there are limitations to this review. First, this is a narrative review. As more data becomes available in this rapidly evolving field then a systematic review may be valuable. Secondly, many cohort studies have not analysed COPD patients as subgroups. It is well recognised that COPD is a highly heterogeneous disease with several clinical phenotypes and a spectrum of severities. Future analysis of cohort studies should attempt to subgroup patients, including by ethnicity, to identify those most at risk.

\section{Conclusion}

This review focused on three key issues concerning COVID-19 and COPD (figure 5). First, it is well known that COPD patients are prone to viral exacerbations $[13,14,16]$, and current evidence shows that COPD patients have increased pulmonary expression of ACE2, the SARS-CoV-2 receptor, providing a mechanism by which COPD patients may be more susceptible to COVID-19 [41]. COPD patients also demonstrate features of endothelial cell dysfunction and increased coagulopathy, which may predispose to increased risk of worse outcomes from COVID-19 [55-58]. Secondly, there is no clinical evidence that ICS are protective against COVID-19 or are associated with worse clinical outcomes [94]. Finally, whilst the available evidence from cohort studies does not demonstrate that COPD patients are more or less susceptible to acquiring infection with SARS-CoV-2, clinical outcomes including requirement for mechanical ventilation and mortality appear to be worse in COPD patients [102-107, 111].

Conflict of interest: A. Higham reports personal fees from Chiesi, outside the submitted work. A. Mathioudakis reports grants from Boehringer Ingelheim outside the submitted work. J. Vestbo reports personal fees from AstraZeneca, Boehringer-Ingelheim, Chiesi, GSK and Novartis, and grants from Boehringer-Ingelheim, outside the submitted work. D. Singh reports personal fees from AstraZeneca, Boehringer Ingelheim, Chiesi, Cipla, Genentech, GlaxoSmithKline, Glenmark, Gossamerbio, Menarini, Mundipharma, Novartis, Peptinnovate, Pfizer, Pulmatrix, Theravance and Verona, outside the submitted work.

Support statement: This research was supported by the NIHR Manchester Biomedical Research Centre and the North West Lung Centre Charity, Manchester. This report is independent research and the views expressed in this publication are those of the authors and not necessarily those of the NHS, the NIHR or the Dept of Health. Funding information for this article has been deposited with the Crossref Funder Registry. 


\section{References}

1 Centre for Health Protection of the Hong Kong Special Administrative Region Government. CHP closely monitors cluster of pneumonia cases on Mainland. www.info.gov.hk/gia/general/201912/31/P2019123100667.htm Date last updated: 31 December 2019; date last accessed: 12 June 2020.

2 Chen N, Zhou M, Dong X, et al. Epidemiological and clinical characteristics of 99 cases of 2019 novel coronavirus pneumonia in Wuhan, China: a descriptive study. Lancet 2020; 395: 507-513.

3 Huang C, Wang Y, Li X, et al. Clinical features of patients infected with 2019 novel coronavirus in Wuhan, China. Lancet 2020; 395: 497-506.

4 Zhou F, Yu T, Du R, et al. Clinical course and risk factors for mortality of adult inpatients with COVID-19 in Wuhan, China: a retrospective cohort study. Lancet 2020; 395: 1054-1062.

5 Wang Y, Lu X, Li Y, et al. Clinical course and outcomes of 344 intensive care patients with COVID-19. Am J Respir Crit Care Med 2020; 201: 1430-1434.

6 Wu C, Chen X, Cai Y, et al. Risk factors associated with acute respiratory distress syndrome and death in patients with coronavirus disease 2019 pneumonia in Wuhan, China. JAMA Intern Med 2020; 80: 934-943.

7 Yang $\mathrm{X}, \mathrm{Yu} \mathrm{Y,} \mathrm{Xu} \mathrm{J,} \mathrm{et} \mathrm{al.} \mathrm{Clinical} \mathrm{course} \mathrm{and} \mathrm{outcomes} \mathrm{of} \mathrm{critically} \mathrm{ill} \mathrm{patients} \mathrm{with} \mathrm{SARS-CoV-2} \mathrm{pneumonia} \mathrm{in}$ Wuhan, China: a single-centered, retrospective, observational study. Lancet Respir Med 2020; 8: 475-481.

8 Mehta P, McAuley DF, Brown M, et al. COVID19: consider cytokine storm syndromes and immunosuppression. Lancet 2020; 395: 1033-1034.

9 Gattinoni L, Coppola S, Cressoni M, et al. COVID-19 does not lead to a "typical" acute respiratory distress syndrome. Am J Respir Crit Care Med 2020; 201: 1299-1300.

10 Buja LM, Wolf DA, Zhao B, et al. The emerging spectrum of cardiopulmonary pathology of the coronavirus disease 2019 (COVID-19): report of 3 autopsies from Houston, Texas, and review of autopsy findings from other United States cities. Cardiovasc Pathol 2020; 48: 107233.

11 Varga Z, Flammer AJ, Steiger P, et al. Endothelial cell infection and endotheliitis in COVID-19. Lancet 2020; 395: $1417-1418$.

12 Simonnet A, Chetboun M, Poissy J, et al. High prevalence of obesity in severe acute respiratory syndrome coronavirus-2 (SARS-CoV-2) requiring invasive mechanical ventilation. Obesity (Silver Spring) 2020; 28: 1195-1199.

13 Bafadhel M, McKenna S, Terry S, et al. Acute exacerbations of chronic obstructive pulmonary disease: identification of biologic clusters and their biomarkers. Am J Respir Crit Care Med 2011; 184: 662-671.

14 George SN, Garcha DS, Mackay AJ, et al. Human rhinovirus infection during naturally occurring COPD exacerbations. Eur Respir J 2014; 44: 87-96.

15 Vanfleteren L, Spruit MA, Wouters EFM, et al. Management of chronic obstructive pulmonary disease beyond the lungs. Lancet Respir Med 2016; 4: 911-924.

16 Wilkinson TM, Hurst JR, Perera WR, et al. Effect of interactions between lower airway bacterial and rhinoviral infection in exacerbations of COPD. Chest 2006; 129: 317-324.

17 Mathioudakis AG, Janssens W, Sivapalan P, et al. Acute exacerbations of chronic obstructive pulmonary disease: in search of diagnostic biomarkers and treatable traits. Thorax 2020; 75: 520-527.

18 Shang J, Wan Y, Luo C, et al. Cell entry mechanisms of SARS-CoV-2. Proc Natl Acad Sci USA 2020; 117: 11727-11734.

19 Hoffmann M, Kleine-Weber H, Schroeder S, et al. SARS-CoV-2 cell entry depends on ACE2 and TMPRSS2 and is blocked by a clinically proven protease inhibitor. Cell 2020; 181: 271-80 e8.

20 Li F. Structure, function, and evolution of coronavirus spike proteins. Annu Rev Virol 2016; 3: 237-261.

21 Andersen KG, Rambaut A, Lipkin WI, et al. The proximal origin of SARS-CoV-2. Nat Med 2020; 26: 450-452.

22 Douglas GC, O’Bryan MK, Hedger MP, et al. The novel angiotensin-converting enzyme (ACE) homolog, ACE2, is selectively expressed by adult Leydig cells of the testis. Endocrinology 2004; 145: 4703-4711.

23 Griendling KK, Ushio-Fukai M, Lassegue B, et al. Angiotensin II signaling in vascular smooth muscle. New concepts. Hypertension 1997; 29: 366-373.

24 Lipworth BJ, Dagg KD. Vasoconstrictor effects of angiotensin II on the pulmonary vascular bed. Chest 1994; 105: 1360-1364.

25 Plante GE. Vascular response to stress in health and disease. Metabolism 2002; 51: 25-30.

26 Victorino GP, Newton CR, Curran B. Effect of angiotensin II on microvascular permeability. J Surg Res 2002; 104: 77-81.

27 Brake SJ, Barnsley K, Lu W, et al. Smoking upregulates angiotensin-converting enzyme-2 receptor: a potential adhesion site for novel coronavirus SARS-CoV-2 (Covid-19). J Clin Med 2020; 9: 841.

28 Leung JM, Yang CX, Tam A, et al. ACE-2 expression in the small airway epithelia of smokers and COPD patients: implications for COVID-19. Eur Respir J 2020; 55: 2000688.

29 Zhang H, Rostami MR, Leopold PL, et al. Expression of the SARS-CoV-2 ACE2 receptor in the human airway epithelium. Am J Respir Crit Care Med 2020; 202: 219-229.

30 Ferrario CM, Jessup J, Chappell MC, et al. Effect of angiotensin-converting enzyme inhibition and angiotensin II receptor blockers on cardiac angiotensin-converting enzyme 2. Circulation 2005; 111: 2605-2610.

31 Wang F, Xia ZF, Chen XL, et al. Angiotensin II type-1 receptor antagonist attenuates LPS-induced acute lung injury. Cytokine 2009; 48: 246-253.

32 Imai Y, Kuba K, Rao S, et al. Angiotensin-converting enzyme 2 protects from severe acute lung failure. Nature 2005; 436: 112-116.

33 Sun NN, Yu CH, Pan MX, et al. Mir-21 mediates the inhibitory effect of Ang (1-7) on AngII-induced NLRP3 inflammasome activation by targeting Spry1 in lung fibroblasts. Sci Rep 2017; 7: 14369.

34 Jia HP, Look DC, Shi L, et al. ACE2 receptor expression and severe acute respiratory syndrome coronavirus infection depend on differentiation of human airway epithelia. J Virol 2005; 79: 14614-14621.

35 Benetti E, Tita R, Spiga O, et al. ACE2 gene variants may underlie interindividual variability and susceptibility to COVID-19 in the Italian population. Eur J Hum Genet 2020; 28: 1602-1614.

36 Binkhorst M, Offringa AK, van der Hoeven JG. COVID-19: comprehensive synopsis of suggested pathophysiological mechanisms and repurposed drugs. Preprints 2020; preprint [https://doi.org/10.20944/ preprints202007.0108.v1]. 

proteolysis by TMPRSS2 augments entry driven by the severe acute respiratory syndrome coronavirus spike protein. J Virol 2014; 88: 1293-1307.

38 Glowacka I, Bertram S, Herzog P, et al. Differential downregulation of ACE2 by the spike proteins of severe acute respiratory syndrome coronavirus and human coronavirus NL63. J Virol 2010; 84: 1198-1205.

39 Kuba K, Imai Y, Rao S, et al. A crucial role of angiotensin converting enzyme 2 (ACE2) in SARS coronavirus-induced lung injury. Nat Med 2005; 11: 875-879.

40 Cai G, Bosse Y, Xiao F, et al. Tobacco smoking increases the lung gene expression of ACE2, the receptor of SARS-CoV-2. Am J Respir Crit Care Med 2020; 201: 1557-1559.

41 Smith JC, Sausville EL, Girish V, et al. Cigarette smoke exposure and inflammatory signaling increase the expression of the SARS-CoV-2 receptor ACE2 in the respiratory tract. Dev Cell 2020; 53: 514-529.

42 Radzikowska U, Ding M, Tan G, et al. Distribution of ACE2, CD147, CD26 and other SARS-CoV-2 associated molecules in tissues and immune cells in health and in asthma, COPD, obesity, hypertension, and COVID-19 risk factors. Allergy 2020; in press [https://doi.org/10.1111/all.14429].

43 Higham A, Singh D. Increased ACE2 expression in the bronchial epithelium of COPD patients who are overweight. Obesity (Silver Spring) 2020; 28: 1586-1589.

44 Russo P, Bonassi S, Giacconi R, et al. COVID-19 and smoking. Is nicotine the hidden link? Eur Respir J 2020; 55: 2001116.

45 Leung JM, Yang CX, Sin DD. COVID-19 and nicotine as a mediator of ACE-2. Eur Respir J 2020; 55: 2001261.

46 Ziegler CGK, Allon SJ, Nyquist SK, et al. SARS-CoV-2 receptor ACE2 is an interferon-stimulated gene in human airway epithelial cells and is detected in specific cell subsets across tissues. Cell 2020; 181: 1016-1035.

47 Lukassen S, Chua RL, Trefzer T, et al. SARS-CoV-2 receptor ACE2 and TMPRSS2 are primarily expressed in bronchial transient secretory cells. EMBO J 2020; 39: e105114.

48 Higham A, Quinn AM, Cancado JED, et al. The pathology of small airways disease in COPD: historical aspects and future directions. Respir Res 2019; 20: 49.

49 Gundavarapu S, Wilder JA, Mishra NC, et al. Role of nicotinic receptors and acetylcholine in mucous cell metaplasia, hyperplasia, and airway mucus formation in vitro and in vivo. J Allergy Clin Immunol 2012; 130: 770-780.

50 Pinheiro TA, Barcala-Jorge AS, Andrade JMO, et al. Obesity and malnutrition similarly alter the renin-angiotensin system and inflammation in mice and human adipose. J Nutr Biochem 2017; 48: 74-7482.

51 Hsu AC, Parsons K, Moheimani F, et al. Impaired antiviral stress granule and IFN-beta enhanceosome formation enhances susceptibility to influenza infection in chronic obstructive pulmonary disease epithelium. Am J Respir Cell Mol Biol 2016; 55: 117-127.

52 Geerdink JX, Simons SO, Pike R, et al. Differences in systemic adaptive immunity contribute to the 'frequent exacerbator' COPD phenotype. Respir Res 2016; 17: 140.

53 Kalathil SG, Lugade AA, Pradhan V, et al. T-regulatory cells and programmed death $1+\mathrm{T}$ cells contribute to effector T-cell dysfunction in patients with chronic obstructive pulmonary disease. Am J Respir Crit Care Med 2014; 190: 40-50.

54 Grundy S, Plumb J, Lea S, et al. Down regulation of T cell receptor expression in COPD pulmonary CD8 cells. PLoS One 2013; 8: e71629.

55 Kasahara Y, Tuder RM, Cool CD, et al. Endothelial cell death and decreased expression of vascular endothelial growth factor and vascular endothelial growth factor receptor 2 in emphysema. Am J Resp Crit Care 2001; 163: 737-744.

56 Minakata Y, Nakanishi M, Hirano T, et al. Microvascular hyperpermeability in COPD airways. Thorax 2005; 60: 882 .

57 Husebo GR, Gabazza E, D’Alessandro C, et al. Coagulation markers in COPD. Eur Respir J 2018; 52: Suppl. 62, OA1937.

58 Vaidyula VR, Criner GJ, Grabianowski C, et al. Circulating tissue factor procoagulant activity is elevated in stable moderate to severe chronic obstructive pulmonary disease. Thromb Res 2009; 124: 259-261.

59 Aleva FE, Voets L, Simons SO, et al. Prevalence and localization of pulmonary embolism in unexplained acute exacerbations of COPD: a systematic review and meta-analysis. Chest 2017; 151: 544-554.

60 Vogelmeier CF, Criner GJ, Martinez FJ, et al. Global Strategy for the Diagnosis, Management, and Prevention of Chronic Obstructive Lung Disease 2017 Report: GOLD Executive Summary. Eur Respir J 2017; $49: 1700214$.

61 Soler-Cataluna JJ, Martinez-Garcia MA, Roman Sanchez P, et al. Severe acute exacerbations and mortality in patients with chronic obstructive pulmonary disease. Thorax 2005; 60: 925-931.

62 Halpin DM, Decramer M, Celli B, et al. Exacerbation frequency and course of COPD. Int J Chron Obstruct Pulmon Dis 2012; 7: 653-661.

63 Mallia P, Footitt J, Sotero R, et al. Rhinovirus infection induces degradation of antimicrobial peptides and secondary bacterial infection in chronic obstructive pulmonary disease. Am J Respir Crit Care Med 2012; 186: 1117-1124.

64 Calverley PMA, Anzueto AR, Carter K, et al. Tiotropium and olodaterol in the prevention of chronic obstructive pulmonary disease exacerbations (DYNAGITO): a double-blind, randomised, parallel-group, active-controlled trial. Lancet Respir Med 2018; 6: 337-344.

65 Lipson DA, Barnhart F, Brealey N, et al. Once-daily single-inhaler triple versus dual therapy in patients with COPD. N Engl J Med 2018; 378: 1671-1680.

66 Papi A, Vestbo J, Fabbri L, et al. Extrafine inhaled triple therapy versus dual bronchodilator therapy in chronic obstructive pulmonary disease (TRIBUTE): a double-blind, parallel group, randomised controlled trial. Lancet 2018; 391: 1076-1084.

67 Bafadhel M, Peterson S, De Blas MA, et al. Predictors of exacerbation risk and response to budesonide in patients with chronic obstructive pulmonary disease: a post-hoc analysis of three randomised trials. Lancet Respir Med 2018; 6: 117-126.

68 Pascoe S, Barnes N, Brusselle G, et al. Blood eosinophils and treatment response with triple and dual combination therapy in chronic obstructive pulmonary disease: analysis of the IMPACT trial. Lancet Respir Med 2019; 7: 745-756.

69 Siddiqui SH, Guasconi A, Vestbo J, et al. Blood eosinophils: a biomarker of response to extrafine beclomethasone/formoterol in chronic obstructive pulmonary disease. Am J Respir Crit Care Med 2015; 192: $523-525$. 

controlled trial. Lancet 2017; 389: 1919-1929.

71 Mathioudakis AG, Bikov A, Foden P, et al. Change in blood eosinophils following treatment with inhaled corticosteroids may predict long-term clinical response in COPD. Eur Respir J 2020; 55: 1902119.

72 Agusti A, Fabbri LM, Singh D, et al. Inhaled corticosteroids in COPD: friend or foe? Eur Respir J 2018; 52: 1801219.

73 Singh D, Roche N, Halpin D, et al. Current controversies in the pharmacological treatment of chronic obstructive pulmonary disease. Am J Respir Crit Care Med 2016; 194: 541-549.

74 Singh D, Agusti A, Anzueto A, et al. Global Strategy for the Diagnosis, Management, and Prevention of Chronic Obstructive Lung Disease: the GOLD science committee report 2019. Eur Respir J 2019; 53: 1900164.

75 Glass CK, Ogawa S. Combinatorial roles of nuclear receptors in inflammation and immunity. Nat Rev Immunol 2006; 6: 44-55.

76 Higham A, Karur P, Jackson N, et al. Differential anti-inflammatory effects of budesonide and a p38 MAPK inhibitor AZD7624 on COPD pulmonary cells. Int J Chron Obstruct Pulmon Dis 2018; 13: 1279-1288.

77 Kunz LIZ, Ten Hacken NH, Lapperre TS, et al. Airway inflammation in COPD after long-term withdrawal of inhaled corticosteroids. Eur Respir J 2017; 49: 1600839.

78 Lapperre TS, Snoeck-Stroband JB, Gosman MM, et al. Effect of fluticasone with and without salmeterol on pulmonary outcomes in chronic obstructive pulmonary disease: a randomized trial. Ann Intern Med 2009; 151: 517-527.

79 Contoli M, Pauletti A, Rossi MR, et al. Long-term effects of inhaled corticosteroids on sputum bacterial and vira loads in COPD. Eur Respir J 2017; 50: 1700451.

80 Garcha DS, Thurston SJ, Patel AR, et al. Changes in prevalence and load of airway bacteria using quantitative PCR in stable and exacerbated COPD. Thorax 2012; 67: 1075-1080.

81 Bewley MA, Budd RC, Ryan E, et al. Opsonic phagocytosis in chronic obstructive pulmonary disease is enhanced by Nrf2 agonists. Am J Respir Crit Care Med 2018; 198: 739-750.

82 Higham A, Scott T, Li J, et al. Effects of corticosteroids on COPD lung macrophage phenotype and function. Clin Sci (Lond) 2020; 134: 751-763.

83 Martinez-Garcia MA, Faner R, Oscullo G, et al. Inhaled steroids, circulating eosinophils, chronic airway infection, and pneumonia risk in chronic obstructive pulmonary disease. a network analysis. Am J Respir Crit Care Med 2020; 201: 1078-1085.

84 Singanayagam A, Glanville N, Girkin JL, et al. Corticosteroid suppression of antiviral immunity increases bacterial loads and mucus production in COPD exacerbations. Nat Commun 2018; 9: 2229.

85 Skevaki CL, Christodoulou I, Spyridaki IS, et al. Budesonide and formoterol inhibit inflammatory mediator production by bronchial epithelial cells infected with rhinovirus. Clin Exp Allergy 2009; 39: 1700-1710.

86 Thomas BJ, Porritt RA, Hertzog PJ, et al. Glucocorticosteroids enhance replication of respiratory viruses: effect of adjuvant interferon. Sci Rep 2014; 4: 7176.

87 Singanayagam A, Johnston SL. Long-term impact of inhaled corticosteroid use in asthma and chronic obstructive pulmonary disease (COPD): review of mechanisms that underlie risks. J Allergy Clin Immunol 2020; in press [https://doi.org/10.1016/j.jaci.2019.12.907].

88 Yamaya M, Nishimura H, Deng X, et al. Inhibitory effects of glycopyrronium, formoterol, and budesonide on coronavirus $\mathrm{HCoV}-229 \mathrm{E}$ replication and cytokine production by primary cultures of human nasal and tracheal epithelial cells. Respir Investig 2020; 58: 155-168.

89 Matsuyama S, Kawase $\mathrm{M}$, Nao $\mathrm{N}$, et al. The inhaled corticosteroid ciclesonide blocks coronavirus RNA replication by targeting viral NSP15. bioRxiv 2020; preprint [https://doi.org/10.1101/2020.03.11.987016].

90 Jeon S, Ko M, Lee J, et al. Identification of antiviral drug candidates against SARS-CoV-2 from FDA-approved drugs. Antimicrob Agents Chemother 2020; 64: e00819-20.

91 Deng X, Hackbart M, Mettelman RC, et al. Coronavirus nonstructural protein 15 mediates evasion of dsRNA sensors and limits apoptosis in macrophages. Proc Natl Acad Sci USA 2017; 114: E4251-E4E60.

92 Finney LJ, Glanville N, Farne H, et al. Inhaled corticosteroids downregulate the SARS-CoV-2 receptor ACE2 in COPD through suppression of type I interferon. bioRxiv 2020; preprint [https://doi.org/10.1101/2020.06.13.149039].

93 Peters MC, Sajuthi S, Deford P, et al. COVID-19 related genes in sputum cells in asthma: relationship to demographic features and corticosteroids. Am J Respir Crit Care Med 2020; 202: 83-90.

94 Halpin DMG, Singh D, Hadfield RM. Inhaled corticosteroids and COVID-19: a systematic review and clinical perspective. Eur Respir J 2020; 55: 2001009.

95 Schultze A, Walker AJ, MacKenna B, et al. Inhaled corticosteroid use and risk COVID-19 related death among 966,461 patients with COPD or asthma: an OpenSAFELY analysis. medRxiv 2020; preprint [https://doi.org/10. $1101 / 2020.06 .19 .20135491]$.

96 Chalmers JD, Laska IF, Franssen FME, et al. Withdrawal of inhaled corticosteroids in COPD: a European Respiratory Society guideline. Eur Respir J 2020; 55: 2000351.

97 Abate SM CY, Mantedafro B, Basu B. Prevalence and risk factors of mortality among hospitalized patients with COVID-19: A systematic review and Meta-analysis. Bull World Health Organ 2020; in press [http://dx.doi.org/10. 2471/BLT.20.260737].

98 Alqahtani JS, Oyelade T, Aldhahir AM, et al. Prevalence, severity and mortality associated with COPD and smoking in patients with COVID-19: a rapid systematic review and meta-analysis. PLoS One 2020; 15: e0233147.

99 Argenziano MG, Bruce SL, Slater CL, et al. Characterization and clinical course of 1000 patients with coronavirus disease 2019 in New York: retrospective case series. BMJ 2020; 369: m1996.

100 Guan WJ, Ni ZY, Hu Y, et al. Clinical characteristics of coronavirus disease 2019 in China. N Engl J Med 2020; 382: $1708-1720$.

101 Marcello RK, Dolle J, Grami S, et al. Characteristics and outcomes of COVID-19 patients in New York City's Public Hospital System. medRxiv 2020; preprint [https://doi.org/10.1101/2020.05.29.20086645].

102 Bartoletti M GM, Scudeller L, Tedeschi S, et al. Predictors of severe respiratory failure in hospitalized patients with SARS-CoV-2 infection: development and validation of a prediction model (PREDI-CO study). Clin Microbiol Infect 2020; in press [https://doi.org/10.1016/j.cmi.2020.08.003]. 
Docherty AB, Harrison EM, Green CA, et al. Features of 20133 UK patients in hospital with covid-19 using the ISARIC WHO Clinical Characterisation Protocol: prospective observational cohort study. BMJ 2020; 369 : m1985.

104 Paranjpe I, Russak A, De Freitas JK, et al. Clinical characteristics of hospitalized Covid-19 patients in New York City. medRxiv 2020; preprint [https://doi.org/10.1101/2020.04.19.20062117].

105 Richardson S, Hirsch JS, Narasimhan M, et al. Presenting characteristics, comorbidities, and outcomes among 5700 patients hospitalized with COVID-19 in the New York City area. JAMA 2020; 323: 2052-2059.

106 Cummings MJ, Baldwin MR, Abrams D, et al. Epidemiology, clinical course, and outcomes of critically ill adults with COVID-19 in New York City: a prospective cohort study. Lancet 2020; 395: 1763-1770.

107 Palmieri L, Vanacore N, Donfrancesco C, et al. Clinical characteristics of hospitalized individuals dying with COVID-19 by age group in Italy. J Gerontol A Biol Sci Med Sci 2020; 75: 1796-1800.

108 Halpin DMG, Faner R, Sibila O, et al. Do chronic respiratory diseases or their treatment affect the risk of SARS-CoV-2 infection? Lancet Respir Med 2020; 8: 436-438.

109 Chu DK, Akl EA, Duda S, et al. Physical distancing, face masks, and eye protection to prevent person-to-person transmission of SARS-CoV-2 and COVID-19: a systematic review and meta-analysis. Lancet 2020; 395: $1973-1987$.

110 Farsalinos K, Angelopoulou A, Alexandris N, et al. COVID-19 and the nicotinic cholinergic system. Eur Respir J 2020; 55: 2001589.

111 Tanoira R P, Garcia F P, Romanyk J, et al. Prevalence and risk factors for mortality related to COVID-19 in a severely affected area of Madrid, Spain. medRxiv 2020; preprint [https://doi.org/10.1101/2020.05.25.20112912].

112 Huang Y, Tan C, Wu J, et al. Impact of coronavirus disease 2019 on pulmonary function in early convalescence phase. Respir Res 2020; 21: 163.

113 Zhao YS, Song Y, Li W, et al. Follow-up study of the pulmonary function and related physiological characteristics of COVID-19 survivors three months after recovery. EClinical Medicine 2020; 25: 100463. 